\title{
Incidence and management of anastomotic leakage following laparoscopic prostatectomy with implementation of a new anastomotic technique incorporating posterior bladder neck tailoring
}

\author{
T. Sukkarieh $\cdot$ J. Harmon $\cdot$ F. Penna $\cdot$ R. Parra
}

Received: 2 September 2007 / Accepted: 11 September 2007 / Published online: 9 October 2007

(c) Springer London 2007

\begin{abstract}
In laparoscopic prostatectomies, vesicourethral anastomotic leaks may result in significant morbidity because of the chemical and metabolic derangements created by urine within the peritoneal cavity. To date, minimal data are available on this problem. Herein we present our experience with urine leaks after RALP. Over a period of 24 months, 135 men underwent RALP. Any drainage creatinine greater than two times the serum creatinine was considered as an anastomotic leak. According to our criteria, $20 \%$ of the first 110 patients developed an anastomotic leak. The patients were analyzed in two groups, those with and without leaks. In the two groups, there was no statistically significant difference in age, height, weight, prostate volume and pre-op hemoglobin. The patients with leaks did have higher rate of prior abdominal surgery ( 50 vs. $36 \%$ ), higher average pre-operative PSA values ( 7.6 vs. 6.1 ), higher rates of multiple biopsies (27 vs. $17 \%$ ) and a higher average BMI (29.6 vs. 27.8). Intraoperative differences included an average of $30 \mathrm{~min}$ longer operative time and $66 \mathrm{~cm}^{3}$ higher average EBL in patients with leaks. The transfusion rate was higher in the leak group at 18 vs. $1 \%$ in the no leak group. Recovery tended to be longer in patients with leaks, with hospital stays of an average of 3.6 days longer. The most common indication for prolonged hospitalization was ileus, which $55 \%$ of patients with leaks developed. Management included placing the catheter on mild traction, continuous antibiotics and taking the drain-off suction with caution to monitor the signs of a worsening ileus. In the last 25 patients, we revised our anastomotic technique. We now include posterior tailoring of the bladder neck prior to the
\end{abstract}

T. Sukkarieh $(\varangle) \cdot J$ J. Harmon · F. Penna $\cdot$ R. Parra

Cooper University Hospital, Camden, NJ, USA

e-mail: ts@ cjurology.com vesicourethral anastomosis when the bladder neck is enlarged. This facilitates a water-tight anastomosis. Using this technique, we have yet to see the anastomotic leak. In RALPs, anastomotic leaks can lead to ileus formation and longer hospital stays. These leaks are associated with a higher average blood loss and transfusion rate. Management should focus on prevention. Since we have incorporated posterior bladder neck tailoring with the anastomosis, the problem has been markedly reduced.

Keywords Prostate cancer - Laparoscopy · Robotics . Complications $\cdot$ Anastomotic leakage

\section{Introduction}

Radical retropubic and radical perineal prostatectomy were the mainstay of surgical treatments for clinically localized prostate cancer until the late 1990s when the development of laparoscopic approaches were reported [1,2]. With the open approaches, the peritoneal cavity is not entered, limiting the sequelae of a vesicourethral anastomotic leak. Anastomotic leaks are noted to occur from 3 to 33\% [3-5] when routine cystograms are performed prior to catheter removal, however little more than continued catheter drainage is required for these patients.

With the advent of laparoscopy and robot-assisted laparoscopy, more surgeries for clinically localized prostate cancer are being performed intraperitoneally. Although infrequent, vesicourethral anastomotic leaks may result in significant morbidity. The chemical and metabolic derangements created by urine within the peritoneal cavity may lead to prolonged ileus formation, longer hospital stays, and elevated serum creatinine [6-8]. To date minimal data are available on this problem. Herein we present 
our experience with urine leaks after robot-assisted laparoscopic prostatectomy (RALP).

\section{Materials and methods}

Over a period of 24 months, 135 men underwent RALP for clinically localized prostate cancer. The anastomotic technique employed was the single-knot technique described by van Velthoven [9]. In the first 110 men, when an enlarged bladder neck was encountered it was closed anteriorly when the vesicourethral anastomosis was completed. In our last 25 patients, a posterior bladder neck tailoring stitch was incorporated with the vesicourethral anastomotic suture prior to the anastomosis being performed. This minimizes any size discrepancy between the urethra and the bladder neck and facilitates a watertight anastomosis.

As our routine practice, on the morning of postoperative day 1 , any patient with a surgical drain output greater than $50 \mathrm{~cm}^{3}$ over the previous $8 \mathrm{~h}$ period underwent a creatinine level from the drain. Any drainage creatinine greater than two times the serum creatinine was considered an anastomotic leak. The surgical drain is maintained until the drainage is less than $30 \mathrm{~cm}^{3}$ over an $8 \mathrm{~h}$ period for two consecutive periods, or if a cystogram demonstrates no extravasation. The urethral catheter is maintained for a minimum of 7 days. If anastomotic leakage is demonstrated by a cystogram or suggested by an elevated creatinine level from the surgical drain, the urethral catheter is left indwelling.

\section{Data analysis}

Our prostate cancer database is prospectively maintained for all patients with pathologically confirmed prostate cancer. A subset database is also prospectively maintained for patients undergoing surgery. After the first 110 robot-assisted laparoscopic prostatectomies, a retrospective database review was performed to analyze patients that developed vesicourethral anastomotic leaks versus those that did not develop this complication. The data were analyzed using an independent $T$-test with Levine correction for equal variance assumption violation.

\section{Results}

According to our criteria, 22 of the first 110 patients $(20 \%)$ developed an anastomotic leak. The patients were retrospectively analyzed in two groups, those with and those without anastomotic leaks. In the two groups, there was no statistically significant difference in age, height, weight, prostate volume, and pre-op hemoglobin. The patients with leaks did have a higher rate of prior abdominal surgery (50 vs. 36\%), higher average pre-operative PSA values (7.6 vs. 6.1 ), higher rates of previous biopsies (27 vs. 17\%), and a higher average BMI (29.6 vs. 27.8). Intraoperative differences included an average $40 \mathrm{~min}$ longer operative time and $91 \mathrm{~cm}^{3}$ higher average EBL in patients with leaks. The transfusion rate was higher in the leak group at 18 versus $1 \%$ in the no leak group (see Table 1).

Recovery tended to be longer in patients with leaks, with hospital stays an average of 3.3 days longer. The most common indication for prolonged hospitalization was an ileus, which $55 \%$ of patients with leaks developed. The surgical drain tended to stay in longer in the patients with leaks, for an average of 9.7 versus 1.8 days. Additionally, $68 \%$ of patients with leaks were discharged from the hospital with the surgical drain indwelling versus $3.4 \%$ of patients without (Table 1). Management included placing the catheter on mild traction, continuous antibiotics, and taking the drain off suction with caution to monitor for signs of a worsening ileus.

Management of these leaks should focus on prevention. In patients with enlarged prostates and/or median lobes, an exaggerated bladder neck is often encountered intraoperatively (Fig. 1). In our first 110 cases, when a discrepancy was found between the urethral stump and the bladder neck, the bladder neck was tailored anteriorly once the anastomosis was completed. We have since revised our anastomotic technique and now include posterior tailoring of the bladder neck prior to the vesicourethral anastomosis when the bladder neck is enlarged (Fig. 2). Using a double armed 2-0 monocryl at a total length of $25 \mathrm{~cm}$, the bladder neck is tailored posteriorly (Fig. 3). This allows the ureteral orifices to be placed at a further distance from the vesicourethral anastomosis.

Table 1 Leakage versus no leakage patients

\begin{tabular}{lccc}
\hline & No Leakage & Leakage & $p$ value \\
\hline Number of patients & 88 & 22 & \\
Mean BMI & 27.8 & 29.6 & 0.12 \\
Mean number of prior biopsies & 0.24 & 0.71 & 0.13 \\
Percentage of patients with & 36 & 50 & 0.32 \\
$\quad$ prior surgeries & & & \\
Mean operative time (min) & 198.7 & 238.7 & $0.01 *$ \\
Mean estimated blood loss (cc) & 185 & 276 & $0.02 *$ \\
Transfusion rate (\%) & 1.1 & 18 & $<0.01 *$ \\
Mean days with drain & 1.8 & 9.7 & $<0.01 *$ \\
Percentage of patients & 3.4 & 68 & $<0.01 *$ \\
$\quad$ discharged with drain & & & \\
Mean hospital stay & 1.7 & 4.9 & $<0.01 *$ \\
Mean days with catheter & 10.4 & 13.8 & $<0.01 *$ \\
\hline
\end{tabular}

a Asterisks indicate significant differences between leakage and no leakage groups 


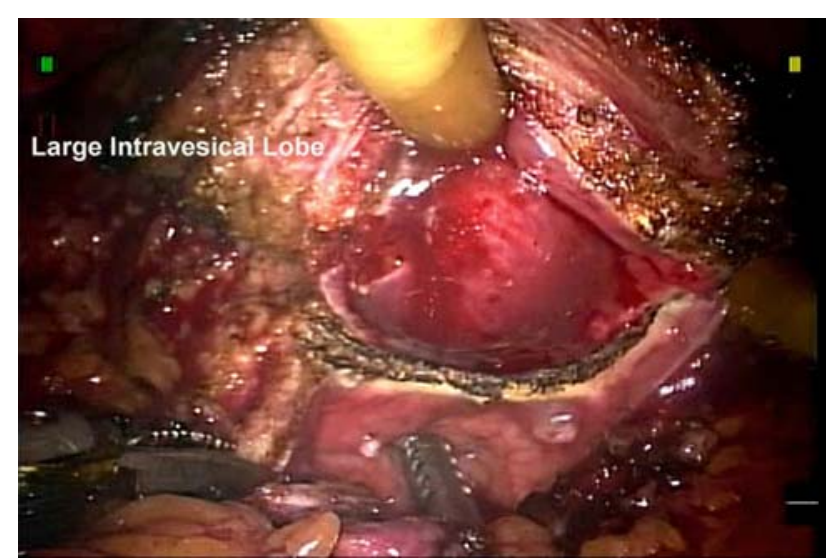

Fig. 1 Large intravesical lobe

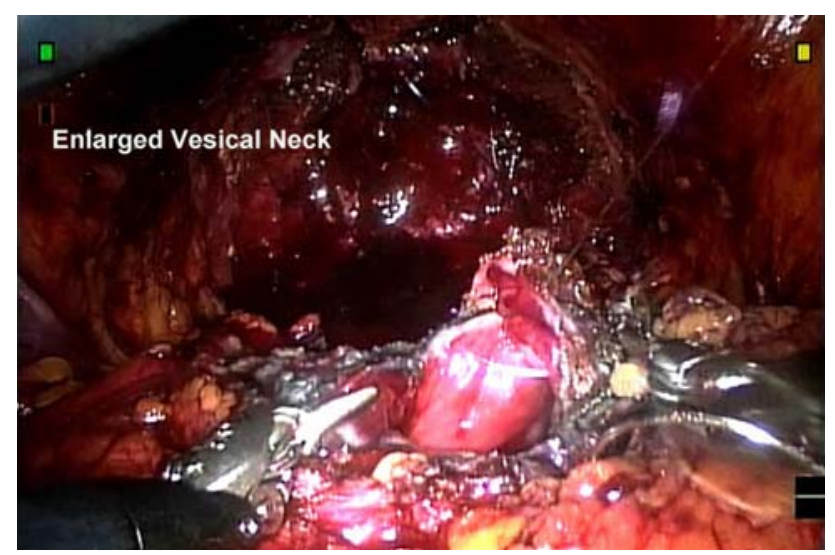

Fig. 2 Enlarged vesical neck

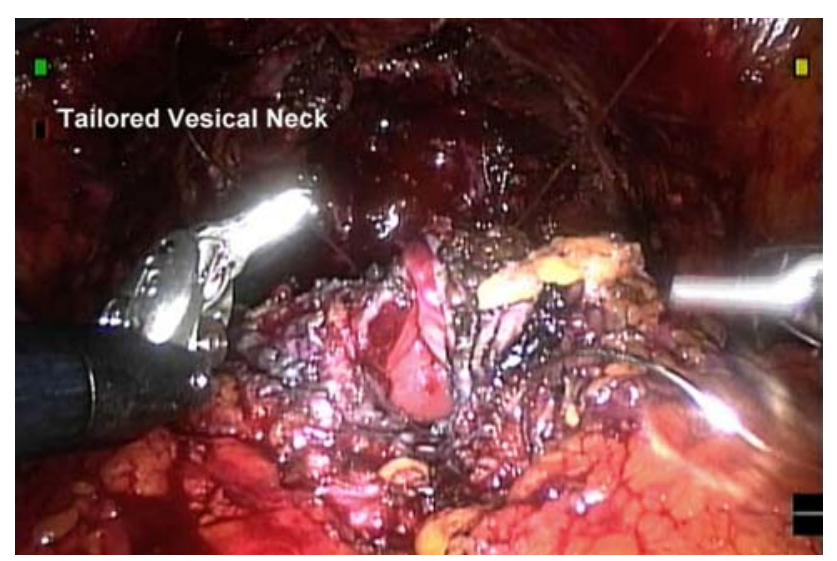

Fig. 3 Tailored vesical neck

With the same stitch, a running continuous vesicourethral anastomosis is performed over a 20 French catheter (Fig. 4). This maneuver facilitates a true water-tight running anastomosis by allowing correction of the size discrepancy between the urethra and bladder neck. With an additional 25 cases to date, we have not seen any further anastomotic leaks since incorporating posterior bladder neck tailoring with the vesicourethral anastomosis.

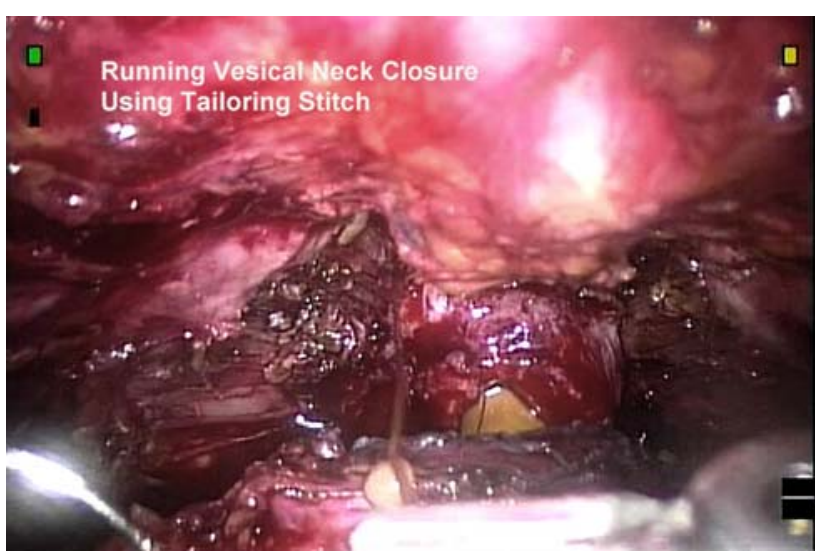

Fig. 4 Running Vesical neck closure using tailoring stitch

\section{Conclusion}

With intraperitoneal access for laparoscopic radical prostatectomy, anastomotic leaks can lead to urine within the peritoneal cavity. This has the potential for chemical and metabolic derangements, particularly for the intraperitoneal cavity exposed to urine. These derangements include prolonged ileus, elevated serum creatinine and longer hospital stays. Vesicourethral anastomotic leaks are associated with a higher average blood loss and transfusion rate. Risk factors may include a higher BMI, prior abdominal surgery, and a history of multiple prostate biopsies.

\section{References}

1. Schuessler WW, Schulam PG, Clayman RV, Kavoussi LR (1997) Laparoscopic radical prostatectomy: initial short-term experience. Urology 50(6):854-857

2. Guillonneau B, Vallancien G (1999) Laparoscopic radical prostatectomy: initial experience and preliminary assessment after 65 operations. Prostate 39(1):71-75

3. Noguchi M, Shimada A, Yahara J, et al (2004) Early catheter removal 3 days after radical retropubic prostatectomy. Int $\mathbf{J}$ Urol 11(11):983-988

4. Levy JB, Ramchandani P, Berlin JW, et al (1994) Vesicourethral healing following radical prostatectomy: is it related to surgical approach? Urology 44(6):888-892

5. Gnanapragasam VJ, Baker P, Naisby GP, Chadwick D (2005) Identification and validation of risk factors for vesicourethral leaks following radical retropubic prostatectomy. Int J Urol 12(11):948-952

6. Pintar TJ, Wilke RA (1998) Urinary ascites: spontaneous bladder rupture presenting as acute oliguric renal failure. Am J Med 105(4):347-349

7. Dees A, Kluchert SA, van Vliet AC (1990) Pseudo-renal failure associated with internal leakage of urine. Neth J Med 37(5-6):197-201

8. Chow KM, Lam CW, Szeto CC (2005) Pseudo-renal failure following total abdominal hysterectomy. J Nephrol 18(4):442-446

9. Van Velthoven RF, Ahlering TE, Peltier A, et al (2003) Technique for laparoscopic running urethrovesical anastomosis: the singleknot method. Urology 61(4):699-702 